\title{
Collected Data Set Size Considerations for Atom Probe Cluster Analysis
}

\author{
Matthew Swenson ${ }^{1}$, Janelle Wharry ${ }^{1}$ \\ 1. Boise State University, Department of Materials Science and Engineering, Boise, ID, U.S.A.
}

Atom probe tomography is increasingly being used to complement transmission electron microscopy (TEM) to characterize microstructures, particularly for nano-featured materials containing phases that are below TEM resolution limits. Local electrode atom probe (LEAP) tomographic cluster analysis algorithms provide an objective means to identify and measure the size and number density of these nano-scale phases. However, there is a lack of standardized methodology for quantifying average cluster size, which presents ambiguities and challenges when attempting to compare nanocluster morphology between different specimens. A critical consideration in LEAP data analysis is the number of ions collected from each needle. Thus, the objective of this study is to consider the effect of LEAP collected sample size on the measured cluster size, and to suggest methods to improve the fidelity of comparing average cluster sizes between larger and smaller data sets.

The alloys selected for study are a commercial ferritic-martensitic (F-M) steel HCM12A and an oxide dispersion strengthened (ODS) steel, both of which are candidates for advanced reactor cladding and structural components [1]. The HCM12A (Fe-10.5Cr-1.43W-1.5Cu-1.04Ni-0.52Mn-0.3Si) is selected because of its tendency to nucleate $\mathrm{Si}-\mathrm{Mn}-\mathrm{Ni}$-P-rich and $\mathrm{Cu}$-rich nanoclusters upon irradiation [2], while the ODS (Fe-8.67Cr-1.95W-0.28Y-0.23Ti) is selected because of its pre-existing distribution of Ti-Y-O nanoclusters, which have exhibited varying degrees of morphological stability under irradiation [3]. Both alloys were irradiated to $3 \mathrm{dpa}$ at $500^{\circ} \mathrm{C}$, using three different particles - neutrons, protons, and $\mathrm{Fe}^{++}$self-ions - for a total of six irradiated samples. Multiple LEAP needles were prepared from each sample using focused ion beam, and each needle was analyzed with a Cameca LEAP 4000X HR. Each LEAP data set was reconstructed with the Integrated Visualization and Analysis Software (IVAS) using the voltage history during the LEAP analysis and was visually inspected to ensure the tip shape was consistent with the SEM image prior to analysis. Cluster analysis was performed on each tip using the maximum separation method, with the parameter $d_{\max }$ and $\mathrm{N}_{\min }$ selected to minimize any spurious detection of clusters [4]. Using the IVAS cluster analysis output data, which includes a radius of gyration $\left(R_{g}\right)$ for each cluster, the Guinier diameter $\left(D_{G}\right)$ for each cluster was determined using [5]:

$$
D_{G}=2 \sqrt{\frac{5}{3}} R_{g}
$$

and then averaged within each LEAP data set (i.e. within each tip).

Both the ODS and HCM12A consistently reveal that for a given sample, the average cluster size (i.e. Guinier diameter) increases with the total amount of ranged ions in each sample (i.e. the relative size of the collected sample), as shown in Figure 1. Our observation can be explained by considering the magnification $(M)$ of the tip reconstruction [6]:

$$
M=\frac{L}{\xi R}
$$

where $L$ is the distance from the sample to the detector, $R$ is the specimen radius of curvature, and $\xi$ is the image compression factor. For each data set reconstruction, $\xi$ was kept consistent $(=1.65)$. Since each sample needle was similarly shaped, the larger samples result in data being progressively collected further away from the detector. Consequently, $L$ is likely increasing more rapidly than $R$, leading to larger magnification of the ion trajectories, and ultimately resulting in inflated sizes of identified clusters 
in the larger data sets.

This magnification effect can potentially lead to misleading conclusions regarding nanocluster size evolution with irradiation, especially if only one needle is collected from each sample. If, for example, in the ODS alloy, the proton- and Fe ion-irradiated needles happened to contain $\sim 15-20$ million ions, while the as-received needle contains $<5$ million ions, the researcher would conclude that oxide nanoclusters experience stability or growth under irradiation. Conversely, if the ODS irradiated needles contain $<5$ million ions, while the as-received needle contains $\sim 15-20$ million ions, the researcher would conclude that irradiation induces cluster dissolution. Similar scenarios can be envisioned for cluster analysis in HCM12A. These potentially contradictory conclusions underscore the need to collect multiple needles from each sample, and to improve the cluster analysis methodology.

We propose one possible approach to improve cluster analysis methodology by comparing samples only at a fixed data set size. If we choose a data set size of 10 million ions, represented by the vertical lines on each plot in Figure 1, linear fit trend lines for all samples tested can be interpolated to this common data set size. Using such an approach would remove any effects of ion trajectory magnification in larger data sets. Future experiments will evaluate the potential to determine if the image compression factor $(\xi)$ may be varied as a function of sample size to offset the magnification effect for larger data sets.

\section{References:}

[1] C. Hin and B. D. Wirth, J. Nucl. Mater. 402 (2010) 30-37.

[2] Z. Jiao and G. S. Was, J. Nucl. Mater. 425 (2012) 105-111.

[3] C.A. Williams et al, Ultramicroscopy 132 (2013) 271-278.

[4] M.J. Swenson and J.P. Wharry, J. Nucl. Mater. 467 (2015) 97-112.

[5] M.K. Miller and R.G. Forbes in "Atom Probe Tomography: The Local Electrode Atom Probe", (Springer, New York, 2014) p. 321.

[6] D.J. Larson et al in "Local Electrode Atom Probe Tomography", (Springer, New York, 2013) p. 120. [7] The authors acknowledge the US Nuclear Regulatory Commission (NRC-HQ-84-14-G-0056), DOE Nuclear Science User Facilities (14-486, 15-540 and 15-569), and J. Burns and Y.Q. Wu at the Center for Advanced Energy Studies (CAES).
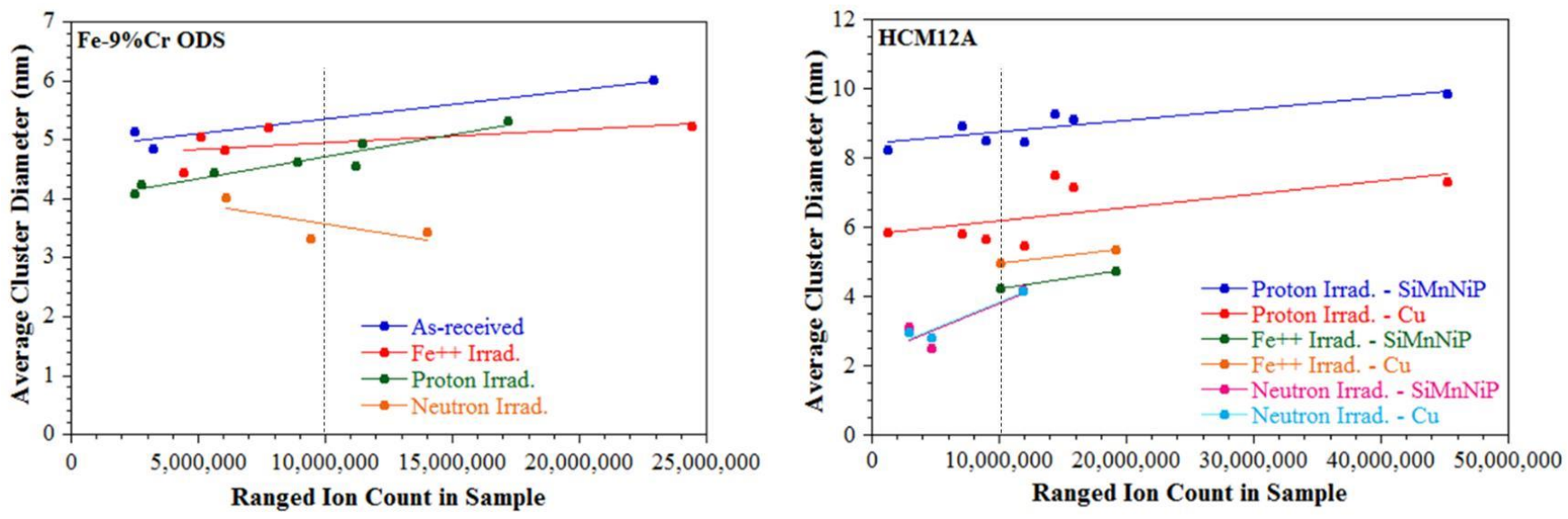

Figure 1. IVAS cluster analysis measurements for average cluster diameter in $\mathrm{Fe}-9 \% \mathrm{Cr}$ ODS and HCM12A. Trend lines demonstrate that cluster size generally increases with collected sample size. 